\title{
Identifying a Carotenoid Cleavage Dioxygenase (CCD4) Gene Controlling Yellow/White Fruit Flesh Color of "Piqiutao" (White Fruit Flesh) and Its Mutant (Yellow Fruit Flesh)
}

\author{
Lu Wen $^{1} \cdot$ Yongqing Wang ${ }^{1} \cdot$ Qunxian Deng ${ }^{1} \cdot$ Min Hong $^{1} \cdot$ Si Shi $^{1} \cdot$ Shanshan He ${ }^{1} \cdot$ Yan Huang ${ }^{1} \cdot$ Hui Zhang $^{1} \cdot$ \\ Cuiping Pan ${ }^{1} \cdot$ Zhiwu Yang $^{1} \cdot{\text { Zhuoheng } \mathrm{Chi}^{1} \cdot \text { Yunmiao Yang }}^{1}$
}

Published online: 25 April 2020

(C) The Author(s) 2020

\begin{abstract}
To better understand the fruit flesh coloration mechanism of peach (Prunus persica), the composition and accumulation of carotenoids were compared, the expression profile of key genes involved in carotenoid biosynthetic and catabolic pathways was performed, and the differentially expressed genes were identified using "Piqiutao" (white fruit flesh) and its mutant yellow "Piqiutao" at different fruit development stages. The results showed that the total carotenoid content in yellow "Piqiutao" was remarkably higher than that of "Piqiutao," and the accumulation of $\beta$-cryptoxanthin, $\alpha$-carotene, and $\beta$-carotene was significantly different, which was most likely caused by the differential expression of $C C D 4$. Therefore, $C C D 4$ may be an essential gene that causes the yellow fruit flesh of yellow "Piqiutao." However, the coding region sequence of CCD4 was entirely identical, and the intron was inserted by a retrotransposon in "Piqiutao" and its mutant, indicating that the expression difference was not caused by the sequence mutation and retrotransposon insertion.
\end{abstract}

Keywords Piqiutao $\cdot$ Mutant $\cdot$ Yellow flesh $\cdot$ Carotenoids $\cdot$ Expression profile $\cdot C C D 4$

Key message This study revealed that the differential expression of $C C D 4$ was likely to be the essential reason for the significant difference in carotenoid content between "Piqiutao" and its yellow-flesh mutant, and the differential expression of $C C D 4$ was not caused by the mutation of the coding region and promoter region of CCD4 and the retrotransposon insertion.
Hui Zhang

510390294@qq.com

Cuiping Pan

524352815@qq.com

Zhiwu Yang

$276577982 @$ qq.com

Zhuoheng Chi

752816158@qq.com

Yunmiao Yang

2286843350@qq.com

College of Horticulture, Sichuan Agricultural University, Chengdu 611130, China 


\section{Introduction}

Peach (Prunus persica) originates from western China and is the third most important temperate fruit tree after apple and pear. Peach flesh color is mainly divided into yellow, red, white, and cyan. The flesh color is a Mendelian genetic trait controlled by a pair of alleles, and white/yellow is controlled by the Y locus of the first linkage group, and white is dominant over yellow (Adami et al. 2013). As compared to white-flesh peach, yellowflesh contains more abundant carotenoids. Carotenoids are mainly a class of $\mathrm{C}_{40}$ terpenoids, which play an essential role in multiple biological processes such as photosynthesis, photomorphogenesis, photoprotection, and development of higher plants (Nisar et al. 2015). Meanwhile, carotenoids are also an indispensable part for human diet health and nutrition. They not only provide humans with important precursors of vitamin A but also help reduce the incidence of cardiovascular diseases, cancer, and blindness (Fiedor and Burda 2014).

Recently, with the development of molecular biology research methods, related genes in carotenoid biosynthesis pathway have been isolated and identified from bacteria and plants, which provides a useful tool for us to utilize genetic engineering to modify carotenoid content and composition. Phytoene synthase (PSY) is the most widely studied enzyme, as it is involved in the key rate-limiting step in the carotenoid synthesis pathway. In tomato fruit, carotenoid biosynthesis pathway is blocked due to activity loss of PSY1, resulting in a yellow mutation, while the missense mutation of PSY2 in cassava causes its roots to appear yellow and accumulate more abundant carotenoids (Welsch et al. 2010; Kachanovsky et al. 2012). Lycopene cyclization is the critical branch of the synthetic pathway, and lycopene $\beta$-cyclase $(\beta$-LCY) and lycopene $\varepsilon$-cyclase $(\varepsilon$-LCY) produce carotenoids with $\beta$-ring and $\varepsilon$-ring, respectively. Linear lycopene produces $\beta$-carotene under LCYB cyclization, whereas LCYE and LCYB act together to synthesize $\alpha$-carotene. The relative expression of $L Y C B$ and $L Y C E$ determines the carotenoid flux, which affects the $\alpha-/ \beta$-carotene ratio (Fu et al. 2019). The $\beta$ carotene content has been greatly improved after silencing $L Y C E$ by RNAi, and the overexpression of $L Y C B 2$ upregulated carotenoid-related genes and increased $\beta$ carotene content in sweet potato (Kim et al. 2013; Kang et al. 2018). Finally, carotenoid cleavage oxygenases (CCOs) catalyze the degradation process of carotenoids, in which carotenoids are cleaved into various apocarotenoids by specifically cutting the conjugated double bond of $\mathrm{C}_{40}$ carotenoids. In Arabidopsis, there are at least nine family members, four CCDs (CCD1, CCD4, CCD7, CCD8), and five NCEDs (NCED2, NCED3, NCED5, NCED6, and NCED9). Carotenoids produce various aromatic substances and phytohormones by CCDs and NCEDs.

Research has increasingly indicated that carotenoid accumulated in various plant species and tissues and was found to be negatively correlated with the expression of $C C D 4$, suggesting its roles in carotenoid turnover. $C C D 4$ gene was highly dynamic, which might contribute to diverse coloration in some plant species; for example, in chrysanthemum, the low expression level of $C m C C D 4 a$ was tightly associated with the loss of the $C m C C D 4 a$ in the genome and the loss of a function that normally enhanced $C m C C D 4 a$ transcription (Yoshioka et al. 2012). In citrus, it was well documented that $C C D 4 b$ was the major factor in determining orange reddish flavedo formation (Zheng et al. 2015). More recently, emerging evidence suggested that the presence of a putative $5^{\prime}$ cisregulatory enhancer within an MITE transposon was responsible for the enhanced allelic expression of $C C D 4 b$ in red-peeled citrus (Zheng et al. 2019). In Arabidopsis, the single nucleotide polymorphisms and insertions and deletions at the locus may be a critical reason for the expression difference of CCD4 (Gonzalez-Jorge et al. 2013). For peach, many researches have shown that $C C D 4$ was a key gene controlling yellow/white trait, and its transcription level in yellow-flesh peaches was closely related to the insertion of a retrotransposon and a frame shift in the microsatellite sequences of the first exon (Fukamatsu et al. 2013). However, the transcription level ultimately determines the number of coding enzyme, which has an important impact on the biochemical process. The activity of the carotenoid metabolism-related enzymes usually is lost in extraction and separation process and is almost inactive in vitro, leading to the failure in accurately measuring their activity. As a result, it is unable to fully confirm the decisive effect of CCD4 on yellow/white trait through biochemical analysis (Giberti et al. 2019). Besides, reported findings are barely focused on the coding region variation of $C C D 4$, which implies that evidences are inadequate and further studies are needed to better understand the fruit flesh coloration mechanism of peach.

In the summer of 2016, a natural mutant with yellow fruit flesh was discovered in "Piqiutao" with white fruit flesh, and the trait was stable in 3 years continuous observation (Fig. 1). These genotypes offer ideal experimental material for the study on fruit flesh coloration mechanism of peach.

In the present study, "Piqiutao" and its yellow mutant were used as research material to reveal the mechanism of coloration mutation from the physiological and molecular aspects, which provided the theoretical basis for the exploitation of carotenoid regulatory genes, the early identification of mutations, and was of great significance for the promotion and application of yellow-flesh peach in the future. 
Fig. 1 Trait comparison between "Piqiutao" (left) and its mutant (right). a Mature fruits of "Piqiutao" (left) and its mutant (right). b Mature flesh of

"Piqiutao" (left) and its mutant (right)
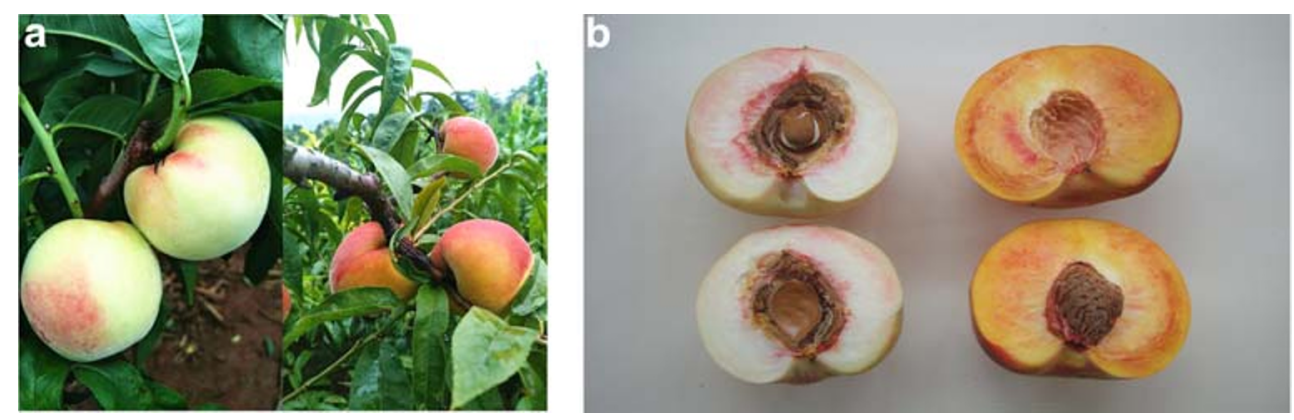

\section{Materials and Methods}

\section{Plant Material and Sample Collection}

"Piqiutao" and its mutant were planted in Wengong Town, Renshou County, Meishan City, Sichuan Province, China. The trees of the two cultivars were 9 years old, whose rootstocks were local wild peach trees. The field management methods, growth situation, and yield were consistent.

Healthy fruits were collected successively at 15-day intervals from May 5, 2017, until July 5 (fruit ripening period). All sample was transferred immediately to the laboratory, and then the pulp was quickly plunged into liquid nitrogen and stored at $-80{ }^{\circ} \mathrm{C}$.

\section{Carotenoid Extraction and Detection}

Carotenoid extraction, carotenoid standard solution preparation, standard curve drawing and HPLC chromatographic conditions referred to the method of Yan et al. (2015), the total carotenoid content was determined by spectrophotometry (Yan et al. 2013a, b). Using Agilent 1260 high performance liquid chromatography system, DAD UV detector and YMCC 30 carotenoid analys is dedicated column $(4.6 \mathrm{~mm} \times 250 \mathrm{~mm}, 5 \mu \mathrm{m})$ were employed to detect the type and amount of carotenoids.

\section{Real-Time Quantitative PCR Analysis of Carotenoid-Related Genes}

The pulp total RNA at different development stages was extracted using a RNAprep Pure Plant Kit from Tiangen Biochemical Technology Corporation, and the first strand of cDNA was synthesized according to the PrimeScriptTM RT reagent Kit (Perfect Real Time) from Takara.

Carotenoid biosynthesis-related genes and reference gene of the peach were synthesized using the primers designed by Brandi et al. (2011), including two genes related to isoprenoid metabolism ( $D X S$ and $H D R$ ), eight genes related to carotenoid biosynthesis (PSY, PDS, ZDS, LYCB, LYCE, ZEP, CHYB, $C H Y E$ ), four genes related to carotenoid metabolism (CCD1, CCD4, NCED1, NCED2), and actin gene (rps28). Real-time
PCR was performed using TaKaRa's TB GreenTM Premix Ex TaqTM II (Tli RNaseH Plus), and each sample was repeated three times. Then, the relative expression of carotenoid related genes was analyzed by $2^{-\Delta \Delta} \mathrm{CT}$ method.

\section{CCD4 Allelic Genotype Verification}

The cDNA of mature "Piqiutao" and its mutant was used as the template to clone the $C C D 4$ coding region sequence. The genome DNA extracted with improved CTAB method was used to verify whether the retrotransposon had been inserted in the intron of CCD4. The PCR amplification primers were listed in Table 1.

\section{Data Processing and Statistical Analysis}

The carotenoid accumulation models and carotenoid synthesis-related gene expression patterns of the pulp of "Piqiutao" and its mutant were represented by GraphPad Prism 5.0. All values were shown as the mean \pm standard errors. Statistical analysis was performed using the SPSS. Student's unpaired $t$ test was used to compare the means at $p<0.05$.

\section{Results and Analysis}

\section{Color Change of Peach Fruit in Different Development Stages}

The flesh color change of "Piqiutao" and its mutant is shown (Fig. 2). In the first phase, the flesh of both

Table 1 PCR amplification primers

\begin{tabular}{ll}
\hline Name & Sequence \\
\hline CCD4-F & GTGAAGGGCAATAC \\
CAGAAT \\
ReD4-R & GGACACATGACACTACAACT \\
Retrotransposon-F & AATACACCATTGAGCGTGAT \\
\hline
\end{tabular}


Fig. 2 Flesh color change of "Piqiutao" (up) and its mutant (down) at different development phases: I, immature green; II, breaker; III, degreening; IV, yellow mature; and V, full mature

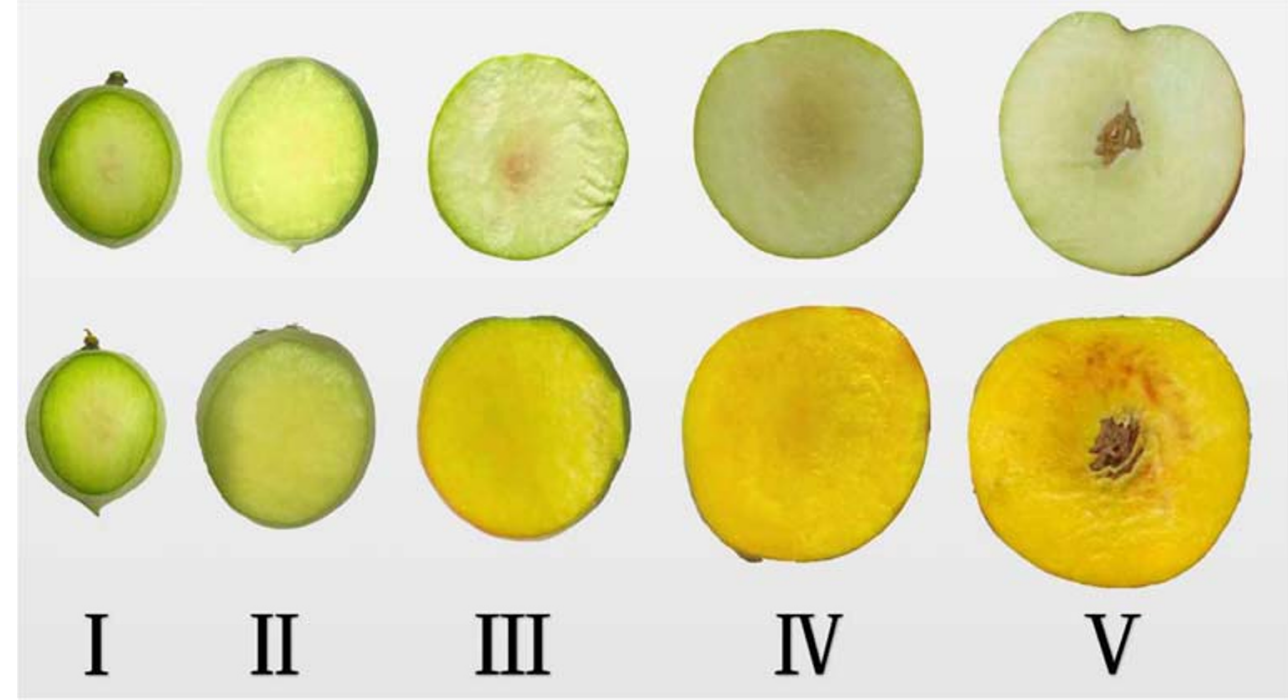

genotypes represented green color, and from the second phase, the flesh color showed coloration difference. "Piqiutao" faded green and gradually turned white, and the mutant turned from green to yellow and the color deepened by degrees.

\section{Carotenoid Accumulation Model}

Carotenoid composition and content analysis in the pulp were analyzed in Fig. 3. It showed that lutein, zeaxanthin, and $\beta$ carotene were predominant in both two cultivars. The content of lutein and zeaxanthin in the two cultivars was roughly similar, and both showed a gradual decline as the fruit matured. The $\beta$-carotene first rose then progressively degraded to disappear in the "Piqiutao," but in the mutant, it gradually reduced to the lowest level and then increased to a maximum of $1.5 \mu \mathrm{g} / \mathrm{gFW}$.

The accumulation pattern of $\beta$-cryptoxanthin and $\alpha$ carotene varied greatly in the two cultivars. At the first stage, $\beta$-cryptoxanthin was both not detected, but in the mid-development stage, the $\beta$-cryptoxanthin content in "Piqiutao" was $0.23 \mu \mathrm{g} / \mathrm{gFW}$ and ultimately disappeared at the mature stage. With regard to mutant, $\beta$ cryptoxanthin began to accumulate from mid-term, whose change tendency was relatively stable, at about $0.28 \mu \mathrm{g} /$ $\mathrm{gFW}$. The $\alpha$-carotene was not detected in "Piqiutao" throughout all stages, whereas the mutant constantly accumulated $\alpha$-carotene from the mid-term and displayed a steady upward trend, reaching the highest content at maturity, about $0.97 \mu \mathrm{g} / \mathrm{gFW}$.

During the fruit ripening process, the total content of carotenoids in the mutant was significantly higher than that in "Piqiutao." As the fruit matured, the total content of carotenoids in "Piqiutao" continuously declined to a minimum of $0.99 \mu \mathrm{g} / \mathrm{gFW}$, while the mutant behaved a gradual upward trend, reaching a maximum content of $14.08 \mu \mathrm{g} / \mathrm{gFW}$ at maturity, which was about 14-fold higher than "Piqiutao."

\section{Carotenoid Synthesis-Related Gene Expression Pattern}

Carotenogenic gene expression pattern in the pulp is shown in Fig. 4. Among these genes, the expression trends of PSY, ZDS, $\angle Y C B, Z E P$, and CHYB of "Piqiutao" coincided with the mutant, and among them, the $Z D S$ expression abundance in mutant was remarkably higher than that of "Piqiutao" throughout all periods. However, the expression profiles of PDS, LYCE, and $C H Y E$ were slightly different. The $P D S$ gradually increased and later decreased in "Piqiutao"; however, in the mutant, it first rose, a decline thereafter, then increased, and finally sharply dropped. $L Y C E$ was slowly up-regulated to third stage and then rapidly decreased to the lowest level in "Piqiutao." As for the mutant, the highest expression abundance period was at the second stage, followed by an abrupt decrease to the lowest level.

NCED1 and NCED2 had a relatively similar dynamic change, both were in a stable low expression state in the early development stage and then rose sharply to the highest point in the late stage, and the expression level of mutant was extremely higher than that of "Piqiutao." CCD1 in both genotypes appeared as a rapid increase, then a slight decrease, an abrupt rise, and an eventual decrease. Nevertheless, the expression pattern of CCD4 was absolutely distinct. "Piqiutao" initially was at low level, then slowly rose from the first stage to the third stage, after that sharply up-regulated to the highest level, and finally slightly decreased at maturity, while CCD4 

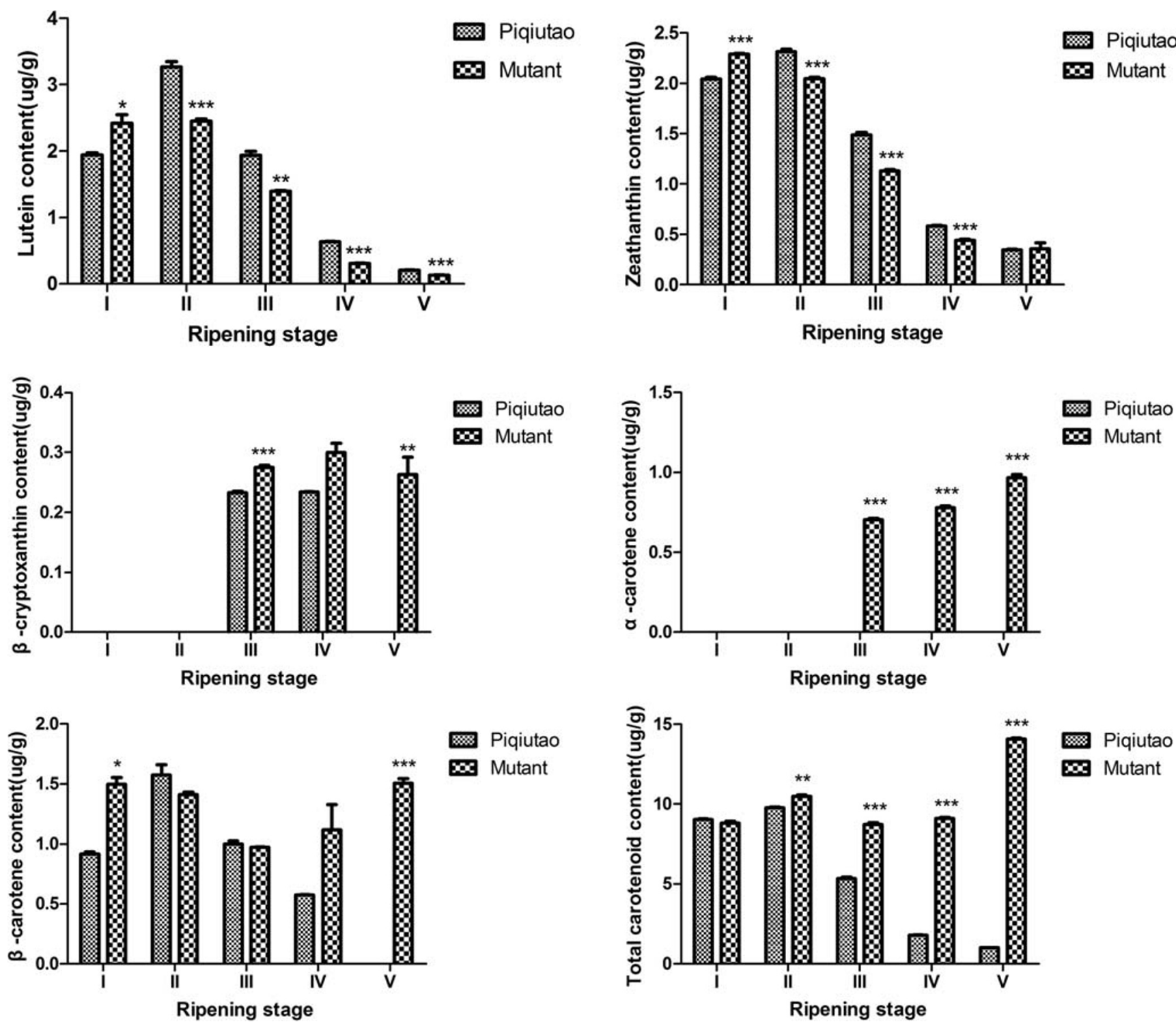

Fig. 3 Carotenoid content and compositions in peach pulp at various developmental stages. I, immature green; II, breaker; III, degreening; IV, yellow mature; and $\mathrm{V}$, full mature. ${ }^{*}$ Significant difference at $p=0.05$

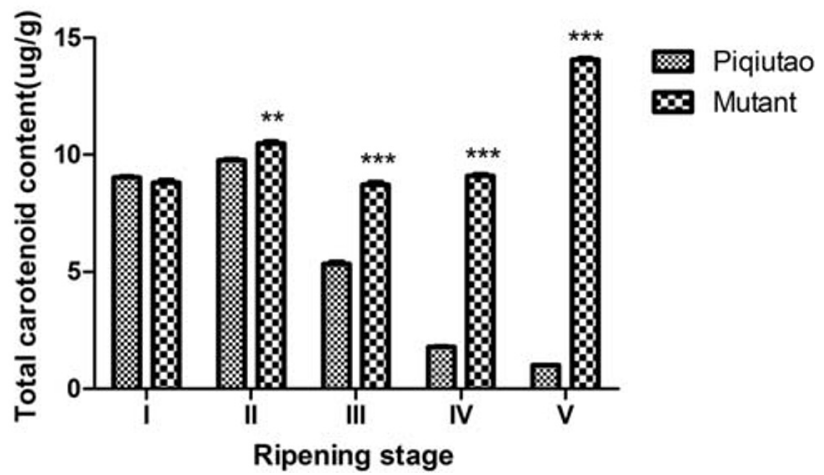

level; **significant difference at $p=0.01$ level; ***significant difference at $p=0.001$ level

was lowly expressed in the mutant, and its abundance was always markedly lower than that of "Piqiutao."

\section{Identification of CCD4 Allelic Genotype}

The $C C D 4$ coding region cloning primers were designed based on 5' UTR and 3' UTR regions, and sequencing results illustrated that the open reading frames (ORF) of CCD4 were $1794 \mathrm{bp}$ in the two peaches, which belonged to the homozygous $\mathrm{w}^{1}$ genotype.

The retrotransposon insertion verification primers were designed based on the first exon sequence and retrotransposon sequence, and the electropherograms indicated that there existed a retrotransposon in the intron of $C C D 4$ of the two cultivars, and both were heterozygous for the $y^{2}$ genotype (Fig. 5).

\section{Discussion}

\section{Accumulation Difference of Carotenoid Leads to the Yellow-Flesh Mutant}

Researches on the peach carotenoid have shown that the primary portions were lutein, zeaxanthin, $\beta$-cryptoxanthin, $\alpha$ carotene, and $\beta$-carotene, in which the $\beta$-carotene content was the highest, and the fruit color is mainly associated with the component and amount of carotenoids (Yan et al. 2013a, 
a
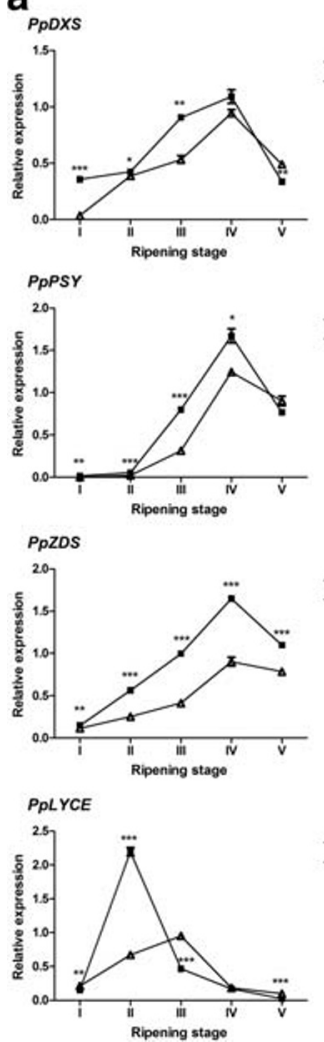

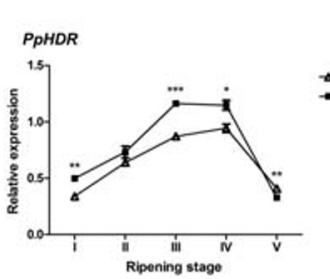

PPPDS

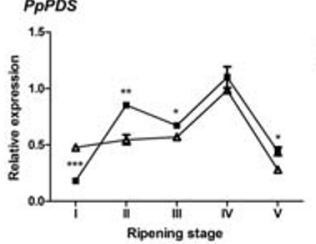

PpLYCB
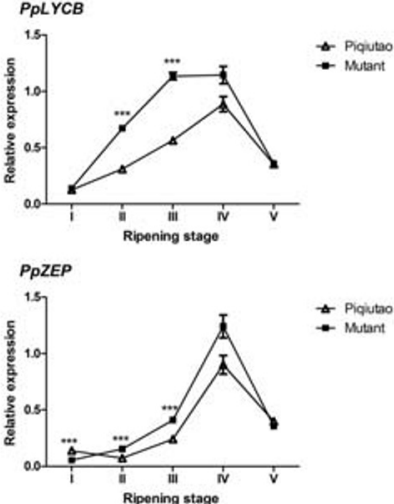

Fig. 4 Expression of key genes in carotenoid biosynthesis and metabolism of peach fruit in different developmental stages. I, immature green; II, breaker; III, degreening; IV, yellow mature; and V,

b). In our study, lutein and zeaxanthin displayed uniform pattern and content in the two cultivars; therefore, they were not the major cause for the color divergence. In the pulp, $\beta$ cryptoxanthin and $\alpha$-carotene started to accumulate from mid-development, and the content in the mutant was significantly higher than that of "Piqiutao." Moreover, the $\beta$ carotene content in the mutant was also obviously higher than "Piqiutao" at maturity; therefore, the continuous accumulation of $\beta$-cryptoxanthin, $\alpha$-carotene, and $\beta$-carotene in the mutant was a critical reason for the color distinction. However, Zhu

a

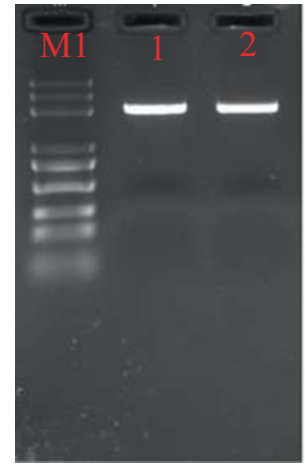

b

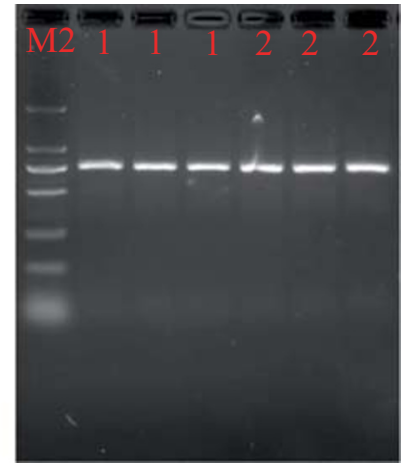

Fig. 5 Retrotransposon electropherogram. a Absence of retrotransposon insertion. b Presence of retrotransposon insertion. 1, "Piqiutao"; 2, mutant; M1, DL5000 marker; M2, DL2000 marker

\section{b}
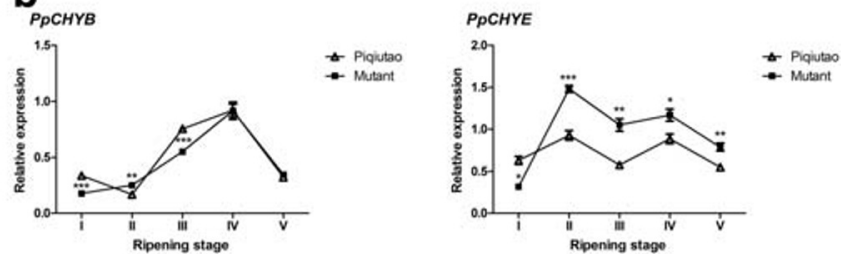

PPNCEDT

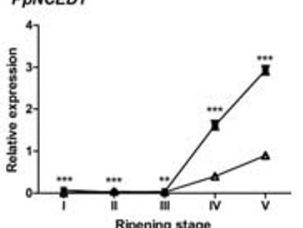

- Piquitao

PPNCED2

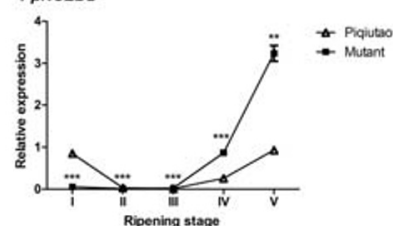

PpCCD1
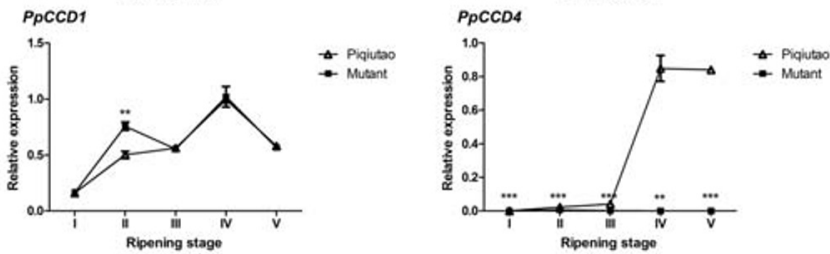

full mature. *Significant difference at $p=0.05$ level; **significant difference at $p=0.01$ level; $* * *$ significant difference at $p=0.001$ level

et al. (2015) considered that the flesh color of "Zhongyoutao 9 " mutant was chiefly associated with the accumulation of violaxanthin, $\beta$-carotene, and zeaxanthin, indicating that the accumulation pattern of carotenoid was diverse in peach varieties.

\section{Inactivation of CCD4 Leads to Carotenoid Accumulation in the Flesh of Mutant}

The abundant accumulation of $\beta$-cryptoxanthin, $\alpha$-carotene, and $\beta$-carotene in the pulp of the mutant may be linked with the joint regulation of $H D R, P S Y, Z D S, L Y C B, L Y C E$, and CHYE. The NCED1 and NCED2 expression patterns were inconsistent with the carotenoids accumulation, but $C C D 4$ diversified completely, which was always low in the mutant and strongly expressed in "Piqiutao" at late stages. These results combined to suggest that the expression difference of $C C D 4$ was the major determinant for the color mutation, which was coherent with the previous researches on the white/yellow pulp coloration mechanism of peach (Adami et al. 2013; Ma et al. 2014; Zhu et al. 2015; Bai et al. 2016; Cao et al. 2017; Zhu et al. 2018).

Higher plants primarily utilize carotenoid dioxygenase (CCD) and 9-cis-epoxy carotenoid dioxygenase (NCED) to 
cleave carotenoids into various apo-carotenoids, and CCD4 possesses the capacity of cleaving carotenoids located in the plastids into colorless substance, for the reason that its transcription levels are usually negatively correlated with carotenoid content (Ruiz-Sola et al. 2014). Song et al. (2017) assumed that the down-regulation of $M d C C D 4 b$ was one of the major determinants for carotenoid accumulation at the ripening stage in the yellow-flesh apple. The CCD4 expression abundance in yellow azalea petals was much lower than that of white azalea petals during the growth process, and the $C p C C D 4 a$ and $C p C C D 4 b$ were highly expressed in whiteflesh summer squash, which was negatively related to carotenoid content, and for wolfberry, CCD4 was highly expressed in petals and leaves, and its expression level was consistent with the $\beta$-carotene accumulation (González-Verdejo et al. 2015; Ureshino et al. 2016; Tian et al. 2017).

\section{CCD4 Multiple Variation Mechanisms in Different Plants}

CCD4 exists multiple mutation mechanisms, leading to expressing distinctly between yellow and white tissues. Studies have discovered that white chrysanthemum mutated to yellow petals owing to the $C m C C D 4 a$ deletion in the genome, by RNAi treatment, white chrysanthemum accumulated large amount carotenoid and turned into yellow petals (Ohmiya et al. 2006; Ohmiya et al. 2009; Jo et al. 2016). The yellow petal formation of Brassica napus was tightly concerned with the function loss of $C C D 4$ as a result of the CACTA-like transposable element insertion in the coding region (Zhang et al. 2015). For Osmanthus fragrans, the differential expression of $C C D 4$ was closely related to its promoter methylation degree and the regulation of OfWRKY3 transcription factor (Han et al. 2014; Han et al. 2016). In peach, there were at least three different variation mechanisms in $P p C C D 4$, including the number difference of microsatellite repeats(TC)n, retrotransposon insertion in intron, and single nucleotide mutation, as a result, CCD4 cannot express normally, causing a yellow-/white-flesh color shift (Falchi et al. 2013). Zhu (2017) revealed that the microsatellite repeat (TC)n number variation played a key role in determining the low expression of $C C D 4$ in the "Zhongyoutao 9" yellow-flesh mutant.

However, in this study, the coding region sequence of $C C D 4$ was entirely identical, and the intron was inserted by a retrotransposon in "Piqiutao" and its mutant, which varied with former studies. The carotenoids accumulation pattern in plant tissues is regulated by various mechanisms, and the final amount is mainly determined by its biosynthesis, metabolism, and storage capacity (Nisar et al. 2015). Carotenoids are synthesized and stored in plastids, which suggested that plastid-related genes are a critical regulatory point for its accumulation levels, such as the Or discovered in cauliflower, the $P A P$ associated with the whiteflesh loquat formation, and fibrillin protein richened in red pepper (Lu et al. 2006; Simkin et al. 2007; Fu et al. 2012). Similarly, in this study, the appearance of yellow-flesh mutant may be partially linked with these genes. Certainly, transcription factor is also one of the important elements regulating the transcription level of CCD4. For example, Han et al. (2016) considered that OfWRKY3 had a positive regulatory effect on $C C D 4$, and the expression divergence of $C C D 4$ was responsible for the color difference in petals of "Dangui" and "Yingui." Meanwhile, Ma et al. (2014) also found that the promoter region of CCD4 contained cis-acting elements associated with photoresponse and hormone response, which may have affected its temporal expression pattern.

\section{Conclusion}

Collectively, these results of this study elucidated that $C C D 4$ expression difference had nothing to do with its sequence mutation and retrotransposon insertion, and there may exist other unknown regulatory mechanisms such as transcription factors and miRNAs. More importantly, results from the above further complemented the previous reported findings, and future investigations on the carotenoid regulation mechanism may contribute to profoundly understand the mutation causes. The discovery and research of the spontaneous mutant are of great significance for exploiting carotenoid regulatory genes and breeding new yellow-flesh peach varieties.

Funding Information This work was funded by the Sichuan Science and Technology Support Project (2016NYZ0-034).

Open Access This article is licensed under a Creative Commons Attribution 4.0 International License, which permits use, sharing, adaptation, distribution and reproduction in any medium or format, as long as you give appropriate credit to the original author(s) and the source, provide a link to the Creative Commons licence, and indicate if changes were made. The images or other third party material in this article are included in the article's Creative Commons licence, unless indicated otherwise in a credit line to the material. If material is not included in the article's Creative Commons licence and your intended use is not permitted by statutory regulation or exceeds the permitted use, you will need to obtain permission directly from the copyright holder. To view a copy of this licence, visit http://creativecommons.org/licenses/by/4.0/.

\section{References}

Adami M, De Franceschi P, Brandi F, Liverani A, Giovannini D, Rosati C, Dondini L, Tartarini S (2013) Identifying a carotenoid cleavage dioxygenase (ccd4) gene controlling yellow/white fruit flesh color of peach. Plant Mol Biol Rep 31:1166-1175

Bai S, Tuan PA, Tatsuki M, Yaegaki H, Ohmiya A, Yamamizo C, Moriguchi T (2016) Knockdown of carotenoid cleavage dioxygenase 4 (CCD4) via virus-induced gene silencing confers yellow coloration in peach fruit: evaluation of gene function related to fruit traits. Plant Mol Biol Rep 34:257-264 
Brandi F, Bar E, Mourgues F, Horváth G, Turcsi E, Giuliano G, Liverani A, Tartarini S, Lewinsohn E, Rosati C (2011) Study of 'Redhaven' peach and its white-fleshed mutant suggests a key role of CCD4 carotenoid dioxygenase in carotenoid and norisoprenoid volatile metabolism. BMC Plant Biol 11:24

Cao S, Liang M, Shi L, Shao J, Song C, Bian K, Chen W, Yang Z (2017) Accumulation of carotenoids and expression of carotenogenic genes in peach fruit. Food Chem 214:137-146

Falchi R, Vendramin E, Zanon L, Scalabrin S, Cipriani G, Verde I, Vizzotto G, Morgante M (2013) Three distinct mutational mechanisms acting on a single gene underpin the origin of yellow flesh in peach. Plant J 76:175-187

Fiedor J, Burda K (2014) Potential role of carotenoids as antioxidants in human health and disease. Nutrients 6:466-488

Fu X, Kong W, Peng G, Zhou J, Azam M, Xu C, Grierson D, Chen K (2012) Plastid structure and carotenogenic gene expression in red- and whitefleshed loquat (Eriobotrya japonica) fruits. J Exp Bot 63:341-354

Fu X, Cheng S, Feng C, Kang M, Huang B, Jiang Y, Duan X, Grierson D, Yang Z (2019) Lycopene cyclases determine high $\alpha-/ \beta$-carotene ratio and increased carotenoids in bananas ripening at high temperatures. Food Chem 283:131-140

Fukamatsu Y, Tamura T, Hihara S, Oda K (2013) Mutations in the CCD4 carotenoid cleavage dioxygenase gene of yellow-flesh peaches. Biosci Biotechnol Biochem 77:2514-2516

Giberti S, Giovannini D, Forlani G (2019) Carotenoid cleavage in chromoplasts of white and yellow-fleshed peach varieties. J Sci Food Agric 99:1795-1803

Gonzalez-Jorge S, Ha SH, Magallanes-Lundback M, Gilliland LU, Zhou A, Lipka AE, Nguyen YN, Angelovici R, Lin H, Cepela J, Little H, Buell CR, Gore MA, Dellapenna D (2013) CAROTENOID CLEAVAGE DIOXYGENASE4 is a negative regulator of $\beta$-carotene content in Arabidopsis seeds. Plant Cell 25:4812-4826

González-Verdejo CI, Obrero Á, Román B, Gómez P (2015) Expression profile of carotenoid cleavage dioxygenase genes in summer squash (Cucurbita pepo L.). Plant Foods for Hum Nutr 70:200-206

Han Y, Wang X, Chen W, Dong M, Yuan W, Liu X, Shang F (2014) Differential expression of carotenoid-related genes determines diversified carotenoid coloration in flower petal of Osmanthus fragrans. Tree Genet Genomes 10:329-338

Han Y, Wu M, Cao L, Yuan W, Dong M, Wang X, Chen W, Shang F (2016) Characterization of OfWRKY3, a transcription factor that positively regulates the carotenoid cleavage dioxygenase gene OfCCD4 in Osmanthus fragrans. Plant Mol Biol 91:485-496

Jo YD, Kim Y, Ryu J, Choi H, Kim SW, Kang HS, Ahn J, Kim J, Kang S, Kim SH (2016) Deletion of carotenoid cleavage dioxygenase 4a (CmCCD4a) and global up-regulation of plastid protein-coding genes in a mutant chrysanthemum cultivar producing yellow petals. Sci Hortic 212:49-59

Kachanovsky DE, Filler S, Isaacson T, Hirschberg J (2012) Epistasis in tomato color mutations involves regulation of phytoene synthase $1 \mathrm{ex}$ pression by cis-carotenoids. Proc Natl Acad Sci 109:19021-19026

Kang C, Zhai H, Xue L, Zhao N, He S, Liu Q (2018) A lycopene $\beta$ cyclase gene, IbLCYB2, enhances carotenoid contents and abiotic stress tolerance in transgenic sweetpotato. Plant Sci 272:243-254

Kim SH, Kim YH, Ahn YO, Ahn MJ, Jeong JC, Lee HS, Kwak SS (2013) Downregulation of the lycopene epsilon-cyclase gene increases carotenoid synthesis via the beta-branch-specific pathway and enhances salt-stress tolerance in sweetpotato transgenic calli. Physiol Plant 147:432-442

Lu S, Van Eck J, Zhou X et al (2006) The cauliflower or gene encodes a DnaJ cysteine-rich domain-containing protein that mediates high levels of $\beta$-carotene accumulation. Plant Cell Online 18:3594-3605

Ma J, Li J, Zhao J, Zhou H, Ren F, Wang L, Gu C, Liao L, Han Y (2014) Inactivation of a gene encoding carotenoid cleavage dioxygenase (CCD4) leads to carotenoid-based yellow coloration of fruit flesh and leaf Midvein in peach. Plant Mol Biol Rep 32:246-257
Nisar N, Li L, Lu S, Khin NC, Pogson BJ (2015) Carotenoid metabolism in plants. Mol Plant 8:68-82

Ohmiya A, Kishimoto S, Aida R, Yoshioka S, Sumitomo K (2006) Carotenoid cleavage Dioxygenase (CmCCD4a) contributes to white color formation in Chrysanthemum petals. Plant Physiol 142:1193-1201

Ohmiya A, Sumitomo K, Aida R (2009) "Yellow Jimba": suppression of carotenoid cleavage dioxygenase (CmCCD4a) expression turns white Chrysanthemum petals yellow. J Jpn Soc Hortic Sci 78:450-455

Ruiz-Sola MÁ, Arbona V, Gómez-Cadenas A, Rodríguez-Concepción M, Rodríguez-Villalón A (2014) A root specific induction of carotenoid biosynthesis contributes to ABA production upon salt stress in Arabidopsis. PLoS One 9:e90765

Simkin AJ, Gaffé J, Alcaraz J, Carde J, Bramley PM, Fraser PD, Kuntz M (2007) Fibrillin influence on plastid ultrastructure and pigment content in tomato fruit. Phytochemistry 68:1545-1556

Song CX, Zhang LY, Zhang CX, Tian Y, Cong PH (2017) Differential expression analysis of the fruits of yellow-fleshed apple and exploits genes involved in carotenoid pigmentation. Acta Hortic Sin 44:205-214

Tian X, Ji J, Wang G, Jin C, Jia C, Li Z (2017) Cloning and functional characterisation of carotenoid cleavage dioxygenase 4 from wolfberry. J Tianjin Univ 23:62-69

Ureshino K, Nakayama M, Miyajima I (2016) Contribution made by the carotenoid cleavage dioxygenase 4 gene to yellow colour fade in azalea petals. Euphytica 207:401-417

Welsch R, Arango J, Bär C, Salazar B, Al-Babili S, Beltrán J, Chavarriaga P, Ceballos H, Tohme J, Beyer P (2010) Provitamin a accumulation in cassava (Manihot esculenta) roots driven by a single nucleotide polymorphism in a phytoene synthase gene. Plant Cell 22:3348-3356

Yan SB, Cai ZX, Yu ML, Zhang YY, Ma RJ (2013a) Incarnadine formation and carotenoids changes in peach fruit at different development stages. Acta Bot-Occid Sin 33:613-619

Yan SB, Zhang YY, Ma RJ, Yu ML (2013b) Changes of carotenoids composition of yellow peach during fruit development. J Fruit Sci 30:260-266

Yan J, Cai ZX, Shen ZJ, Zhang BB, Ma RJ, Yu ML (2015) Extraction and analytical methods of carotenoids in fruit of yellow flesh peach. J Fruit Sci 32:1267-1274

Yoshioka S, Aida R, Yamamizo C, Shibata M, Ohmiya A (2012) The carotenoid cleavage dioxygenase 4 (CmCCD4a) gene family encodes a key regulator of petal color mutation in chrysanthemum. Euphytica 184:377-387

Zhang B, Liu C, Wang Y, Yao X, Wang F, Wu J, King GJ, Liu K (2015) Disruption of a carotenoid cleavage dioxygenase 4 gene converts flower colour from white to yellow in Brassica species. New Phytol 206:1513-1526

Zheng XJ, Xie ZZ, Zhu KJ, Xu Q, Deng XX, Pan ZY (2015) Isolation and characterization of carotenoid cleavage dioxygenase 4 genes from different citrus species. Mol Gen Genomics 290:1589-1603

Zheng XJ, Zhu KJ, Sun Q, Zhang WY, Wang X, Cao HB, Tan ML, Xie ZZ, Zeng YL, Ye JL, Chai LJ, Xu Q, Pan ZY, Xiao SY, Fraser PD, Deng XX (2019) Natural variation in CCD4 promoter underpins speciesspecific evolution of red coloration in citrus peel. Mol Plant 1-14

Zhu YQ (2017) Identification of yellow-flesh mutant of 'CN9' nectarine and analysis of gene expression related to carotenoid metabolism, vol. doctor (p. 125). Chinese Academy of Agricultural Sciences dissertation

Zhu YQ, Zeng WF, Lu ZH, Niu L, Cui GC, Wang ZX (2015) Carotenoid metabolism and gene expression analysis of 'CN9' nectarine and its yellow flesh mutant 'CN9Y'. Acta Hortic Sin 42:623-632

Zhu YQ, Zeng WF, Niu L, Cai ZG, Lu ZH, Pan L, Cui GC, Wang ZX (2018) Temporal and spatial expression of carotenoid metabolism and relative genes in flowers and leaves of CN9 nectarine and its yellow-fleshed mutant. Acta Hortic Sin 45:1869-1880

Publisher's Note Springer Nature remains neutral with regard to jurisdictional claims in published maps and institutional affiliations. 